\title{
El XIV Plan Quinquenal 2021-2025: reto para el nuevo modelo de desarrollo económico de China
}

\author{
XIV Social Economic Development Plan 2021- \\ 2025: Challenges for the New Model of Economic \\ Development of China
}

DOI: $10.32870 /$ mycp.v10i30.742

\author{
Xuedong $\operatorname{Liu}^{1}$ \\ Juan González García
}

\begin{abstract}
Resumen
A partir de la teoría del desarrollo económico, en este artículo se analiza la propuesta de "circulación dual" del nuevo modelo de desarrollo económico chino, sugerido para la formulación del XIV Plan de Desarrollo Económico y Social 2021-2025. El trabajo se realiza desde una doble perspectiva: una retrospectiva que es una revisión del statu quo de la evolución económica china desde hace 40 años; y una prospectiva, que analiza cómo la guerra comercial con Estados Unidos y la Covid-19 le han reforzado y también motu proprio, ajustar su modelo de desarrollo económico, y, en consecuencia, las proyecciones a futuro. La hipótesis que se plantea es que la nueva estrategia, "circulación dual”, le permitirá alcanzar sus objetivos de desarrollo económico tanto de mediano como de largo plazo. Por ello, el artículo espera contribuir al debate teórico, ante la urgencia de buscar alternativas para que los países subdesarrollados logren su anhelado objetivo de desarrollo económico.
\end{abstract}

Palabras clave: estrategia, desarrollo económico, planificación, circulación dual, reforma.

\begin{abstract}
This article analyses the renewed economic development strategy called dual circulations in China from the points of view of the economic development theories, proposed for the XIV Social Economic Development Plan 2021-2025. The analytical methodology is carried out by two perspectives: a retrospective, which reviews the Chinese economic evolution status quo during the last 40 years, and the prospective one, that shows how the trading frictions between China and the United States and Covid-19 have reinforced and as the self-conscious, the Asia country, to adjust its economic development model and the future economic growth projections. It is hypothesized that China will achieve its economic development goals not only in the medium but in the long term through the application of the dual circulations' orientation. For this reason, the work will hope to have a contribution to the theoretical debates before the urgency for the underdeveloped nations to look for alternatives with the purpose to fulfill the desired economic development objective.
\end{abstract}

Keywords: strategy, economic development, plan, dual circulations, reform.

Artículo recibido el 07 de enero de 2021 y dictaminado el 18 de marzo de 2021.

1. Universidad Nacional Autónoma de México (UNAM), Facultad de Estudios Superiores Aragón. Av. Rancho Seco s/n, Nezahualcóyotl, C. P. 57130, Estado de México, México. ORcid: https://orcid. org/0000-0003-2623-4641. Correo electrónico: xdong@comunidad.unam.mx

2. Universidad de Colima, Facultad de Economía-cueicP. Av. Gonzalo de Sandoval 444, Col. Las Víboras, C. P. 28040, Colima, Colima, México. ORciD: https://orcid.org/0000-0003-1458-8047. Correo electrónico: jgogar@ucol.mx 


\section{Introducción}

El inicio de la tercera década del presente siglo representa el punto de partida de la nueva etapa del modelo de desarrollo económico chino a largo plazo, el cual busca consolidar la transformación estructural iniciada a finales de la década de los setenta del siglo $\mathrm{xx}$, y al mismo tiempo dar un impulso adicional del crecimiento económico para los próximos 30 años. Como se puede apreciar, si bien entre 1980 y 2000 China estableció los fundamentos de su modelo de desarrollo económico, en el periodo 2001-2020 experimentó una profundización de éste e hizo ajustes pertinentes al final del lapso ante los indicios cada vez más evidentes de su agotamiento. Esto, sin duda, se ha visto agravado adicionalmente por los efectos generados por la guerra comercial sostenida con Estados Unidos (EU) y por el impacto económico de la Covid-19 recientemente.

Para lograr sus objetivos de cambio estructural y de consolidar su desarrollo económico, China deberá instrumentar un conjunto de acciones y estrategias de políticas socioeconómicas que le permitan alcanzar sus metas económicas con una perspectiva de largo plazo, las cuales deben plasmarse en el XIV Plan Quinquenal de Desarrollo Económico y Social (PQDEYS), 2021-2025.

Es decir, los desequilibrios que le significaron la crisis económica internacional de 2009 y otros eventos ocurridos con anterioridad en materia de crecimiento económico, se acentuaron con la guerra comercial con EU a partir de mediados de 2018 y la Covid-19 en 2020, lo que le han reforzado y también motu proprio a reformular su modelo de desarrollo económico.

De hecho, China ha respondido con diversas estrategias en la última década para hacer frente a los desafíos y a las dificultades generados en los entornos poco alentadores tanto internos como externos, desde la formulación del "sueño chino" y la "nueva normalidad económica", hasta el inicio de las reformas del lado de la oferta.

Asimismo, también ha insistido en su compromiso de llevar a cabo una mayor apertura económica por medio de la Ruta de la Seda (BRI, por sus siglas en inglés) que se ha transformado en una herramienta efectiva y estratégica de integración económica y comercial con el resto del mundo, más el recientemente firmado Regional Comprehensive Economic Partnership (RCEP) con 14 países de la región asiática y del Pacífico.

Por su parte, la propagación de la Covid-19 durante 2020 generó un panorama más complejo desde el ángulo del crecimiento económico, esto a 
pesar de que la economía china se encuentra en una situación privilegiada que le permitió esquivar la recesión en la que cayeron la mayoría de los países del mundo (Fondo Monetario Internacional [FMI], 2021). En este contexto, en 2021 inicia la vigencia el XIV PQDEYS, 2021-2025, plan del que se espera contenga un nuevo esquema de políticas de desarrollo económico.

Internamente, la economía china ha realizado ajustes estructurales de manera significativa desde 2010 con un mercado interno cada vez más fortalecido. Por un lado, el sector manufacturero y el comercio internacional han perdido importancia en relación con el producto interno bruto (PIB). Por otro, las actividades relacionadas con el sector terciario y el componente del consumo doméstico han cobrado cada día más importancia que antes para impulsar el crecimiento económico. En este sentido, una parte considerable del impacto negativo generado por la incertidumbre y los sucesos adversos ocurridos al nivel internacional ha sido compensado por el dinamismo registrado en el consumo doméstico y el sector servicios.

De lo anterior, la hipótesis que se formula en este artículo es que para que China adopte y consolide un nuevo modelo del desarrollo económico, deberá diseñar una estrategia de desarrollo basada en las interacciones de los mercados tanto interno como externo, que priorice la demanda interna. El objetivo del artículo es analizar los ajustes de la nueva política del desarrollo social y económico, cuyo enfoque central consiste en soportar el crecimiento económico con base en el mercado interno complementado con el externo.

En la primera sección se describen los cambios en la política de desarrollo puesta en práctica en el último decenio; en la segunda se analizan algunos aspectos relevantes del nuevo PQDEYS 2021-2025. En la tercera se analizan las implicaciones para las economías, china y mundial, derivadas de la implementación de este nuevo modelo.

\section{Antecedentes de la política económica instrumentada entre 2011 y 2020}

En la década 2011-2020 China implementó un conjunto de acciones para fortalecer aún más su reforma económica y apertura externa, las cuales le permitieron convertirse y consolidarse como la principal potencia comercial del mundo, la segunda economía del orbe, la principal nación subdesarrollada en captar inversión extranjera directa (IED), convertirse en la fábrica del mundo y el mayor productor de diversos bienes a nivel global (González \& Liu, 
2019). Sin embargo, al registrar una tasa de crecimiento económico menor a dos dígitos, se hizo evidente la ralentización de la economía, ya que en los últimos 10 años la tasa de crecimiento económico osciló entre 6 y $7 \%$ (figura 1 ).

\section{Figura 1}

China: tasa de crecimiento económico anual, 2000-2020 (\%)

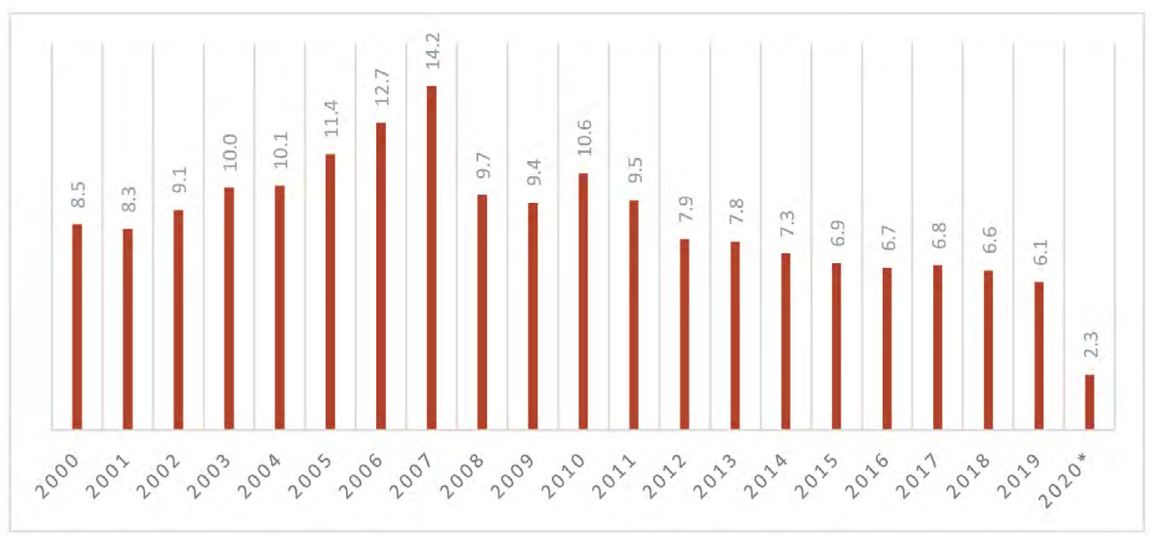

Nota: ${ }^{*}$ preliminar.

Fuente: elaboración propia con base en el National Bureau of Statistics of China (2020a, 2021).

Lo anterior significa que en la década 2011-2020 el crecimiento económico perdió cerca de tres puntos porcentuales (7.7\% contra $10.3 \%$ ) respecto al promedio del decenio anterior, con excepción del subperiodo 2015-2018 que registró un crecimiento cercano a 7\%, que no pudo sostenerse por la desaceleración generada por las fricciones comerciales escalonadas con Estados Unidos (Liu, 2019b).

Por ello, el crecimiento económico del 5.7\% que registró China en el periodo 2016-2020 estuvo lejano del 6.5\% proyectado inicialmente para el periodo del XIII PQDEYS, 2016-2020. Obviamente, gran parte de la explicación del incumplimiento en la meta se debió a la guerra comercial, primeramente $y$, en segundo lugar y de mayor impacto, a la Covid-19. De no ser por estos factores exógenos, China habría logrado el objetivo de crecimiento económico del XIII PQDEYS. 
Ante este panorama, las autoridades económicas chinas han llamado a los agentes económicos a efectuar mayores esfuerzos para superar la ralentización económica. También han tomado múltiples medidas para tratar de conservar el ritmo del crecimiento económico y de esta manera garantizar la creación de empleos. Entre los ajustes de política económica y de desarrollo, destacan el sueño chino, la nueva normalidad económica y la reforma desde el lado de la oferta (Liu, 2019a).

\subsection{El surgimiento del "sueño chino"}

El sueño chino es un término asociado a las diversas estrategias de desarrollo económico, planteadas a partir de las medidas de reforma aplicadas al final de los años setenta. Dicho sueño es la aspiración centenaria de la sociedad y del Gobierno chino de convertir al país en una sociedad con un nivel de desarrollo económico modestamente acomodado en el siglo XXI.

De acuerdo con el planteamiento hecho inicialmente por el entonces máximo líder de este nuevo modelo de desarrollo económico, Deng Xiaoping en 1987, China solo alcanzaría dicho estatus social si lograba un desarrollo integral y la modernización plena, la cual pretendía realizarse a través de tres fases concretas. La primera consistía en la superación del hambre que había padecido la nación, y que fue lograda en los años ochenta del siglo xx.

La segunda inició también en la década de los ochenta y finalizó en 2000 con la cuadruplicación del producto interno bruto (PIB) per cápita, al aumentar desde $\$ 250$ dólares a $\$ 1,000$ dólares, con lo cual el nivel de vida de la población alcanzaría un nivel de desarrollo mediadamente acomodado (Xiaokang). ${ }^{3}$

La tercera etapa, que se inició en el año 2000 y terminará en 2050, corresponde al periodo de despegue importante de la economía china, y que involucra dos "metas centenares". En efecto, cuando el presidente Xi Jinping presentó ante el xIX Congreso Nacional del Partido Comunista Chino (PCCh)

3. Xiaokang es un concepto de tradición milenaria que consiste en una sociedad igualitaria deseada por el confucianismo. En 1979, Deng Xiaoping replanteó este término para describir el proceso de la modernización china; posteriormente en 1984 lo detalló aún más al considerar la sociedad Xiaokang relacionándola con el PIB per cápita de $\$ 800$ dólares al finalizar 2000. De acuerdo con esta visión, Xiaokang, que era una meta sencilla en aquel entonces, sin embargo en la celebración de las V sesiones plenarias del 15vo y 18vo congresos de PCCH en 2000 y 2015, respectivamente, se reforzó la idea de construir y terminar construyendo una sociedad de Xiaokang (medianamente acomodada) de manera integral. 
inaugurado el 18 de octubre de 2017 en el XVIII Comité Central del PCCh, un informe titulado: "Por el logro del triunfo definitivo en la culminación de la construcción integral de una sociedad modestamente acomodada y por la conquista de la gran victoria del socialismo con peculiaridades chinas de la nueva época" (Xinhua 2017), se mencionó con detalle la primera etapa al cumplir los 100 años del nacimiento del Partido Comunista Chino (PCCh) en 2021.

Por ello, para los próximos 30 años todavía se divide en dos fases. En la primera subetapa, en 2021-2035 se pondrán los cimientos para construir una sociedad modestamente acomodada. En la segunda, 2036-2050, se trabajará para convertir a China en un país socialista moderno, próspero, fuerte, democrático, culturalmente avanzado y armonioso (Qiu, 2017). De tal forma, se espera alcanzar la segunda meta centenaria cuando se celebrarán los primeros cien años del establecimiento de la República Popular de China.

De lo anterior se concluye que el sueño chino no es un concepto nuevo, ya que está relacionado con las metas planteadas anteriormente tanto por Deng como por sus sucesores al iniciar este nuevo milenio. Para convertir el sueño chino en realidad, China requiere redoblar esfuerzos, lo que no será fácil ante el panorama económico que priva en la actualidad.

\subsection{La nueva normalidad económica}

Bajo un contexto de desaceleración económica en los últimos 10 años, en mayo de 2014 el presidente Xi pronunció por primera vez el término de la nueva normalidad económica en una visita realizada a la provincia de Henan. Posteriormente se replanteó la misma frase en noviembre del mismo año, en el marco de la reunión cumbre del Foro de Cooperación Económica de Asia Pacífico (APEC, por sus siglas en inglés), celebrada en Beijing en 2014, la cual se sintetiza con los siguientes puntos principales (Sina Finance, 2014):

- El crecimiento económico se encuentra en una etapa de transformación al pasar de una tasa anual de dos dígitos a otra de uno solo, de alrededor de $7 \%$ anual.

- La estructura económica se ajustará a niveles de mayor equilibrio y balance sectorial, enfatizando la importancia de las actividades terciarias con mayor dinámica y participación dentro de la economía, a raíz de la fortaleza y dinámica registradas en el consumo doméstico y el mercado interno. 
- La innovación tecnológica se convertirá en el principal motor del crecimiento económico de manera paulatina y ordenada, en lugar de depender excesivamente del aumento de insumos productivos y de IED.

Por lo planteado, la nueva normalidad económica se refiere al ámbito económico, cuyo ritmo del crecimiento ya se encuentra en una fase de consolidación, después de experimentar por muchos años tasas superiores a dos dígitos. Por ello, su planteamiento no debería catalogarse como bueno o malo, para justificar una tasa particular del crecimiento económico.

No obstante, lo anterior de ninguna manera significaría asumir una actitud conformista en sentido de que ya no se actúa de manera positiva, para propiciar la expansión continua del PIB. China, en tanto como un país en desarrollo aún requiere mantener un crecimiento económico de entre 6 y $7 \%$ para crear el empleo que requiere la población. ${ }^{4}$

Posteriormente, en el contexto del xIII Plan Quinquenal, 2016-2020, el eje central del modelo de desarrollo económico siguió siendo el desarrollo como la tarea primordial. Traducido en porcentajes, la tasa de crecimiento se formuló en un promedio de 6.5\%, el cual permitiría alcanzar en 2020 un ingreso por persona de alrededor de \$10 mil dólares. Ello significó duplicar tanto el PIB como los ingresos per cápita en comparación con los registrados en 2010 (Ríos, 2016).

\subsection{Reformas del lado de la oferta}

Pese a las medidas instrumentadas para enfrentar la desaceleración de la economía, la situación de ralentización ha persistido todavía, tal como se observa en la figura 1 . Al mismo tiempo han surgido nuevos desafíos que impedirían la sustentabilidad del crecimiento económico y que potencialmente propiciarían una mayor desaceleración económica. En primer lugar, existen algunos sectores, como el inmobiliario, con inventarios excesivos. En segundo lugar, varias ramas de la llamada industria pesada, por ejemplo, la producción de cemento, metalúrgica, etc., no solamente registran capacidad

4. Antes de celebrar la tercera sesión del XVIII Comité Central del PCCh en noviembre de 2013, el primer ministro Li enfatizó que el fin del crecimiento económico es asegurar los puestos del trabajo y su creación, por ello se requiere una tasa del crecimiento económico al menos de $7.2 \%$; el reporte puede consultarse en Li, 2013, http://www.china.org.cn/chinese/2013-11/13/content_30582185.htm 
instalada superior a la demanda del mercado, sino que se trata de emisores importantes de gases de efecto invernadero, que deterioran la calidad del aire, agua y el suelo de China.

Por lo anterior, la iniciativa de "Reforma del lado de la oferta" se planteó al final de 2015 e inicios de 2016 previo a la celebración de las XI y XII sesiones plenarias del Partido Comunista Chino. El objetivo de esta acción es ajustar la estructura económica y las funciones del sector público entre los distintos niveles del Gobierno, para facilitar el funcionamiento eficiente del mercado en la asignación de los recursos.

Con estas reformas del lado de la oferta se buscan cambios estructurales a través de medidas de reforma que pretenden: 1) disminuir la capacidad productiva en los sectores poco eficientes y de gran emisión de contaminantes con el propósito de reasignar los recursos a actividades prioritarias y con alto contenido del valor agregado; 2) mejorar la adaptabilidad y flexibilidad de sectores productivos de acuerdo con los hábitos cambiantes y diversificados de los consumidores; 3) aumentar la productividad total de los factores productivos para formar un sistema óptimo de oferta. En pocas palabras, con estas acciones se pretende modificar y regularizar las funciones del Gobierno de acuerdo con las necesidades de las libres fuerzas del mercado, así como lograr la asignación eficiente de los recursos productivos (An, 2016).

En la práctica, desde 2016 se han planteado las siguientes tareas prioritarias para impulsar el crecimiento económico y adecuar la planta productiva, guiada por el mercado tanto nacional como internacional:

- Eliminar el exceso de capacidad productiva instalada en los sectores altamente contaminantes, con bajo nivel de rendimiento; por ejemplo, la producción metalúrgica, mineral de carbón, cemento, etcétera.

- Reducir hasta erradicar el exceso de inventarios que tienen sobreoferta en el mercado, sobre todo las viviendas construidas, que estaban ociosas 
y desocupadas en las ciudades de mediano tamaño en los municipios con una jerarquía administrativa de prefectura hacia abajo ${ }^{5}$ (Liu, 2017).

- Bajar el nivel de apalancamiento del sector financiero, para evitar riesgos y crisis financiera.

- Disminuir los costos, sobre todo los relacionados con la administración pública, que afectan la eficiencia del sector productivo y distorsionan el funcionamiento del mercado.

- Fomentar los sectores atrasados y pendientes por desarrollarse, que concretamente se refieren a la infraestructura y los servicios básicos, que se encuentran con deficiencias en las áreas urbanas.

\subsection{Medidas para impulsar la integración de China a la economía mundial}

Además de lo anterior, China, con el propósito de mantener e incentivar el crecimiento económico ante un entorno - tanto interno como externopoco alentador durante el último decenio, ha aplicado múltiples medidas para impulsar la apertura económica y comercial con mayor profundidad y amplitud. Entre estos nuevos esquemas destacan: el establecimiento de zonas de libre comercio (zonas francas) dentro del territorio chino; el lanzamiento de la iniciativa para formar el Banco Asiático de Inversión en Infraestructura (BAII); los esfuerzos realizados para promover la inclusión de la moneda china en los derechos especiales de giro (DEG) como reserva del Fondo Monetario Internacional (FMI), así como el planteamiento de la BRI como una política general para guiar este proceso de apertura económica y de impulsar la globalización económica (FMI, 2015a, 2015b).

Como es bien sabido, en China la primera zona de libre comercio experimental surgió en 2013 al anunciarse su establecimiento en la ciudad de Shanghái, con el objetivo de formar un área económica que adoptara las reglas internacionales de operación para incrementar su competitividad y relevancia

5. Actualmente existe un total de seis megaciudades, cuatro se incluyen directamente bajo control del Gobierno central y cuentan como entidad provincial: Beijing, Shanghái, Tianjin y Chongqim, y otras dos ciudades cuyo número de población rebasa los 10 millones de habitantes: Shenzhen y Guangzhou. Por su parte, las ciudades capitales de las entidades provinciales suman un total de 28; ciudades de la siguiente jerarquía administrativa (tipo prefectura) con un total de 283; y finalmente son ciudades al nivel de condados y otras numerosas con un rango administrativo todavía inferior que se denomina Zhen, cuyo número de habitantes es de aproximadamente 20 mil personas por localidad con un total de alrededor de 20 mil ciudades. 
a nivel mundial. Para 2020 se han acumulado un total de 21 áreas de libre comercio que cubren el mismo número de provincias de China, es decir, se incrementaron a 20 las áreas de libre comercio en un lapso de siete años. Esto gracias a que en 2020 se tomó la decisión de establecer tres zonas nuevas y se distribuyeron de manera equitativa entre la ciudad de Beijing y las provincias de Hunan y Anhui, respectivamente (Consejo Estatal de China, 2020).

En lo referente a la creación del BAII, éste se concretó en 2015 con el propósito de financiar los proyectos derivados de la BRI principalmente. Hasta la fecha ya cuenta con 103 Estados miembros a nivel mundial e incluye a todos los continentes con excepción de la Antártida. Desde 2016, la entidad financiera de cooperación multilateral ha aprobado más de 80 proyectos y su inversión total hasta la fecha ha superado los 19 mil millones de dólares (Xinhua, 2020a).

Por otra parte, es importante señalar que al $1^{\circ}$ de octubre de 2016 el yuan renminbi (RMB) se convirtió en parte de la canasta de los DEG, con lo que se oficializó como la quinta moneda dentro de las reservas internacionales del FMI, uniéndose al dólar estadounidense, el euro de la Unión Europea, la libra esterlina de la Gran Bretaña y el yen japonés.

Cabe mencionar que cada DEG está compuesto por participaciones respectivas de acuerdo con la importancia que representa cada una de las cinco monedas basada en las participaciones del comercio internacional de las economías involucradas. Actualmente, la moneda china cuenta con una participación de $10.92 \%$, después del dólar de Estados Unidos (41.73\%), del euro (30.93\%); y superior a la del yen (9.33\%) y de la libra (8.09\%) (FMI, 2015a).

Para impulsar este proceso, además de las medidas instrumentadas, el Banco Popular de China impulsó políticas y medidas relacionadas con la emisión de RMB electrónico, iniciando con un plan piloto en las ciudades de Shenzhen, Chengdu y Suzhou, así como la nueva área de Xiongan al final de 2020 (Ministerio del Comercio de China, 2020). Se espera que, de acuerdo con el avance de la prueba piloto, se amplíe a otras regiones, lo cual podría dar un empujón adicional al proceso de la internacionalización del RMB.

En resumen, a pesar de la desaceleración del crecimiento económico, China no ha bajado la guardia ni ha renunciado a sus metas de desarrollo económico plasmadas en sus planes quinquenales, pues entre los dos lapsos respectivos de 2001-2010 y 2011-2020, el PIB en términos de dólares estadounidenses a precios de 2010 se duplicó respectivamente, tal como se contempló en sus programas de desarrollo. Al concluir 2020, se espera que 
supere los 12 billones de dólares, y en moneda local rebase los 101 billones de RMB a precios corrientes.

\section{XIV PQDEYS, 2021-2025: nuevo modelo de desarrollo económico orientado al mercado interno}

Al terminar 2020, el XIII PQDEYS culminó exitosamente, no obstante el impacto de la Covid-19. A partir de 2021 empieza una nueva fase para arrancar el XIV PQDEYS 2021-2025 con el cual se espera cumplir la expectativa de que el país ingrese al club de los países con ingreso alto, de acuerdo con el pronunciamiento realizado al día 3 de noviembre de 2020 por el presidente Xi, al presentar las propuestas del Comité Central del PCch para formular el XIV PQDEYS, 2021-2025 y los objetivos a largo plazo para 2035 (Xinhua, 2020b).

En este sentido, el cumplimiento de las metas del XIV PQDEYS 2021-2025 y los objetivos a largo plazo para 2035 requiere una estrategia novedosa que permita asegurar que el crecimiento económico ya no dependa principalmente del mercado global; en su lugar, el mercado y consumo interno habrán de desempeñar un rol medular y protagónico.

Bajo esta nueva arquitectura, se contempla que la dinámica de la economía se base en el mercado interno complementado por el mercado global, de tal manera que "las interacciones entre los dos tipos de mercado formarán un esquema renovado" para dar un nuevo impulso al crecimiento económico (Xinhua 2020b).

\subsection{Fundamentación teórica para la iniciativa del esquema renovado}

Teóricamente, dependiendo del propósito de la producción de bienes y servicios y de su destino final, se podrían formular distintos esquemas o modelos de crecimiento económico hacia el mercado interno o el externo. Si la producción se orienta hacia el mercado internacional, y el crecimiento económico depende de las exportaciones, el modelo se considera orientado hacia el exterior.

Por su parte, si la producción se dirige a satisfacer las demandas del mercado interno, sustituyendo las importaciones, la estrategia se denomina como de crecimiento orientado hacia el interior, tal como el modelo de sustitución de importaciones que se aplicó en América Latina en el siglo pasado (Villarreal, 1988). De acuerdo con esta perspectiva teórica, el esquema que se ha 
venido aplicando desde hace casi cuatro décadas en China, indudablemente es catalogado como uno de crecimiento hacia el exterior.

Recientemente se han formulado planteamientos de gran relevancia tanto por las autoridades como por los empresarios y los académicos de China, argumentando que el entorno externo ha registrado ajustes importantes durante los últimos años, sobre todo ante la persistencia de los conflictos comerciales entre las dos economías más importantes del mundo actual y la propagación de la Covid-19 en 2020 (Xinhua, 2020b).

Por otro lado, el uso excesivo de los recursos productivos ha evidenciado la insostenibilidad del crecimiento económico de depender mayoritariamente de la inversión y demanda exterior; igualmente, la creciente incertidumbre y riesgo que han surgido y prevalecido en los mercados internacionales, también han vulnerado la aplicabilidad del modelo vigente.

En esta lógica, seguir con la misma estrategia de depender del mercado internacional y de la inversión podría implicar mayores ajustes todavía en su tasa de crecimiento, comprometiendo su prosperidad y sus metas de largo alcance. Derivado de ello, se considera que el crecimiento económico deberá dar un nuevo giro, basándose en la fortaleza y poder de compra del mercado interno.

Este nuevo esquema del crecimiento económico basado en el mercado interno, complementado con el externo, será diferente del modelo que se instrumentó desde hace 40 años. $\mathrm{Al}$ respecto se ha estudiado ampliamente tanto por los propios académicos de aquella nación como por los del resto del mundo y se ha concluido que el éxito económico de China se fundamentó en la dinámica de las exportaciones, que aprovechaban las ventajas comparativas de bajo costo como el salario pagado al factor trabajo y el uso de suelo, entre otros (Liu et al., 2015; González \& Liu, 2019).

No obstante, es importante mencionar que este ajuste en el esquema del crecimiento económico de ninguna manera implicaría que la economía se aislaría de la economía mundial, mucho menos significaría que su propio crecimiento ya tuviera suficiente capacidad para depender solo del mercado doméstico.

Por ello, el nuevo ajuste al modelo de desarrollo económico enfatiza que el crecimiento dependerá de la fortaleza del mercado doméstico y al mismo tiempo se complementa por el mercado global. En otras palabras, las interacciones entre estos dos mercados ${ }^{6}$ seguirán siendo mecanismos para impulsar el

6. En las versiones oficiales, las interacciones entre estos dos mercados se interpretan como "circulación dual" (Xinhua, en vi Magazine, 2020). 
crecimiento económico de China; la gran diferencia consiste en la dominancia de ambos, sin olvidar que en los próximos años el mercado interno tendrá un mayor rol que antes.

\subsection{Ajuste del modelo de crecimiento económico ante un entorno cambiante}

El modelo del crecimiento económico hacia el exterior, sin duda resultó exitoso, y particularmente potenció aún más su dinamismo después de que finalizaron las negociaciones para ingresar a la Organización Mundial del Comercio (OMC) en 2001 (González, 2002), a pesar de que China aceptó que la consideraran como una economía de no mercado, estatus que prevalece hasta la fecha. ${ }^{7}$

A pesar de su éxito relativo, el modelo basado en el mercado global está sujeto a los vaivenes e incertidumbre, además con un costo de explotar los insumos productivos de manera excesiva y agotadora. Por ello, al aproximar sus límites el dinamismo del crecimiento económico tarde o temprano se deterioraría; en consecuencia, requieren de ajustes pertinentes, como el cambio de estrategia.

De hecho, los distintos planteamientos anunciados por las autoridades chinas durante los últimos ocho años, desde el sueño chino, la nueva normalidad económica hasta la reforma del lado de la oferta, justamente han sido reflejo de esta necesidad. En otras palabras, la modificación del modelo de desarrollo económico no es un evento abrupto sino un proceso gradual de prueba y de aprendizaje continuo, que se desencadenó con los acontecimientos surgidos desde 2018, pero sobre todo a partir de 2020.

El crecimiento económico logrado en los últimos 40 años permitió a China no solo convertirse en la segunda economía del mundo, sino también transformarse en múltiples ámbitos, sobre todo con el fortalecimiento del poder adquisitivo de los habitantes $y$, en consecuencia, el potencial creciente del mercado doméstico, lo que se refleja en su contribución al crecimiento económico.

Desde esta perspectiva se han presentado dos sucesos relevantes en la estructura de la economía china: por un lado, a raíz del aumento permanente

7. China sostenía en sus documentos de adhesión a la OMC, que ésta le otorgaría la condición de economía de mercado a partir del 11 de diciembre de 2016. Sin embargo, para muchos otros miembros de la oMc el texto en cuestión —el párrafo 15 del Protocolo de Adhesión de China- está sujeto a otras interpretaciones (Barone, 2015). 
del PIB per cápita y del ingreso disponible de los habitantes, la contribución del consumo y del sector de los servicios se ha incrementado consistentemente en la economía; por otro, se constata que tanto el comercio exterior como la inversión han perdido importancia en su participación respectiva al PIB (figura 2).

\section{Figura 2}

China: contribución al crecimiento por grandes componentes, 2000-2019 (\%)

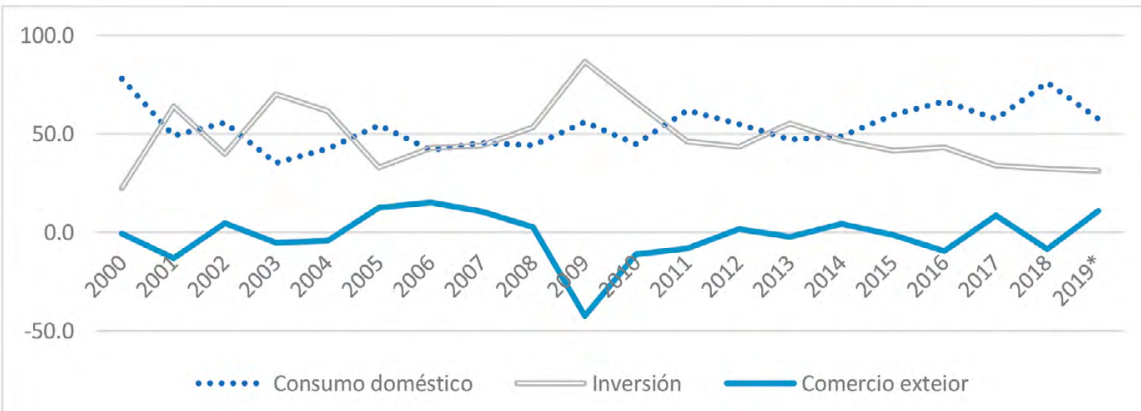

Nota: las cifras de 2019 corresponden a las reveladas en el Reporte anual de desarrollo socioeconómico 2019 publicado por la misma Oficina de Estadísticas de China.

Fuente: elaboración propia con base en el National Bureau of Statistics of China (2020a, 2020b).

En las figuras 2 y 3 se observa que en el presente siglo el consumo doméstico y el sector terciario de la economía registraron un aumento creciente en su contribución al PIB. Si bien en el primer caso, después de que en 2003 cayó hasta su nivel mínimo con el $35.4 \%$, durante los últimos seis años logró elevar su participación en alrededor del 60\%. Por su parte, en 2012 el sector terciario superó por primera vez al sector industrial en su aportación al PIB, desde que inició el proceso de reforma económica y apertura externa de finales de los años setenta del siglo Xx. 


\section{Figura 3}

China: estructura sectorial del PIB, 2000-2019 (\%)

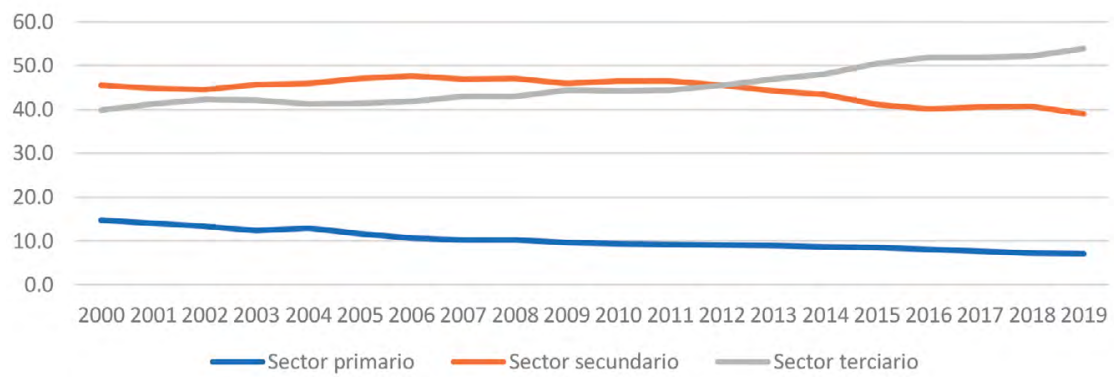

Nota: * las cifras de 2019 corresponden a las reveladas en el Reporte anual de desarrollo socioeconómico 2019 publicado por la misma Oficina de Estadísticas de China.

Fuente: elaboración propia con base en el National Bureau of Statistics of China (2020a, 2020b).

También se aprecia que el grado de dependencia del comercio exterior (la participación de la suma de las exportaciones y las importaciones dentro del PIB) llegó a su punto máximo en 2006 con 64.2\%. Posteriormente la tendencia se revirtió al representar el $31.8 \%$ en 2019, con una reducción del $32.4 \%$; es decir, en 13 años el comercio exterior redujo en más del $50 \%$ su aporte al PIB (figura 4).

\section{Figura 4}

China: grado de dependencia del comercio exterior, 2000-2019 (\%)

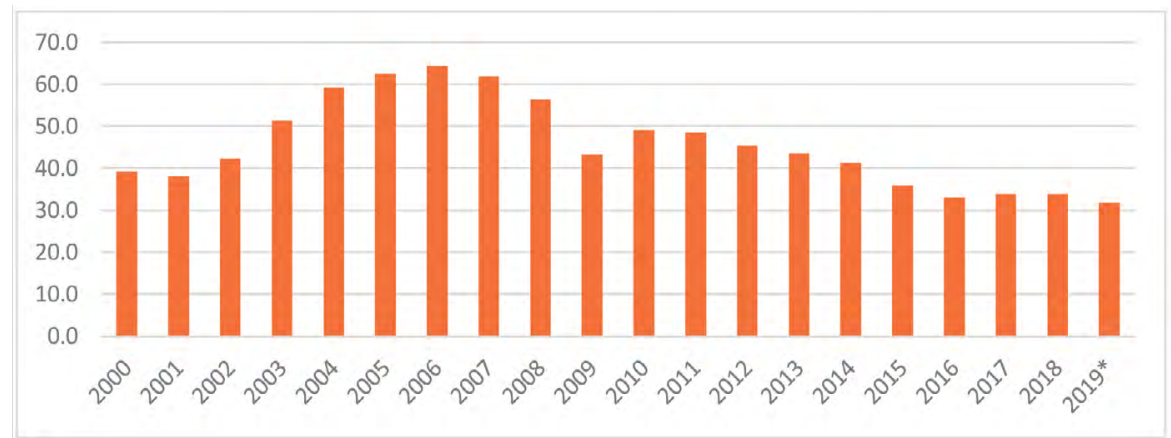

Nota: * las cifras de 2019 corresponden a las reveladas en el Reporte anual de desarrollo socioeconómico 2019 publicado por la misma Oficina de Estadísticas de China.

Fuente: elaboración propia con base en National Bureau of Statistics of China (2020a, 2020b). 
Lo anterior evidenció la creciente incertidumbre y riesgo del sector externo, debido a las decisiones tomadas tanto por los países relevantes en el escenario mundial como por los organismos económicos internacionales. Entre estas decisiones, destacan: la negación de varios miembros de la OMC de otorgar el estatus de economía de mercado a China; el surgimiento del proteccionismo y del unilateralismo del mundo; el inicio y escalonamiento de las fricciones comerciales con Estados Unidos desde mediados de 2018; y recientemente la potencial reconfiguración de las cadenas globales de valor ante la propagación de la Covid-19.

De acuerdo con la versión de las autoridades chinas, su ingreso a la OMC en 2001, condicionado por el estatus de economía de no mercado, se modificaría al finalizar 15 años de vigencia. Es decir, a partir del 11 de diciembre de 2016 los miembros de la OMC, que aún la consideran así, le darían el estatus de economía de mercado. Sin embargo, esto no ha sucedido, debido a las ambigüedades del protocolo de adhesión de China, según las interpretaciones hechas hasta ahora.

De acuerdo con lo estipulado, el miembro de la OMC importador puede utilizar una metodología que no se base en una comparación estricta con los precios internos o los costos en China, si los productores sometidos a investigación no pueden demostrar claramente que en la rama de producción que produce el producto similar prevalecen las condiciones de una economía de mercado en lo que respecta a la manufactura, la producción y la venta de tal producto (párrafo 15 del Protocolo de adhesión de China a la OMC) (Barone, 2015).

Independientemente de que ese párrafo fuera intencionado o no, los esfuerzos realizados por China para cumplir con sus compromisos hechos al momento de su adhesión a la OMC se han cumplido en lo relativo a disminuir las tarifas arancelarias, al pasar de 15.3\% en 2001 a 9.8\% en 2010; adecuación de los diversos reglamentos de acuerdo con los establecidos a nivel internacional, etcétera (The State Council Information Office of the People's Republic of China, 2018). Sin embargo, lo anterior no ha sido reconocido en el marco de la OMC plenamente por algunos miembros, y derivado de ello, el estatus de economía de no mercado lo mantienen algunos países hasta la fecha.

A pesar de ello, China no ha cesado su trabajo en impulsar aún más el proceso de globalización por la vía de buscar acuerdos comerciales regiona- 
les, tal como el recién firmado RCEP. ${ }^{8}$ Por su parte, lo sucedido con el estatus de economía de no mercado ha permitido a China repensar su estrategia de crecimiento económico ante las nuevas circunstancias.

De manera similar, ante el resurgimiento del proteccionismo y del unilateralismo por la llegada del populismo en Estados Unidos, China ha tenido que enfrentar otro reto mayor derivado de los conflictos comerciales y su escalamiento constante desde mediados de 2018. En efecto, además de las fricciones comerciales, el conflicto se ha extendido a otras áreas, como la cooperación tecnológica, la educativa, la protección de la propiedad industrial, etcétera.

Como consecuencia de lo anterior, en el ámbito del intercambio comercial, en su momento más crítico el valor total de los productos chinos sujetos al cobro de aranceles por parte de Estados Unidos alcanzó un monto de \$550 mil millones de dólares, con tasas que van desde 15 a 25\%. En contraparte, China aplicó un nivel similar de sanciones, para un total de $\$ 185$ mil millones de dólares a productos estadounidenses.

El conflicto comercial culminó parcialmente en octubre de 2019 con un acuerdo de primera fase, el cual se firmó el 15 de enero de 2020 por ambas partes, después de haber llevado a cabo un total de 13 rondas de negociaciones. Dentro de lo pactado, se contempla que por parte de Estados Unidos se ajuste la tasa arancelaria para un total de $\$ 120$ mil millones de dólares de mercancías procedentes de China, desde el $15 \%$ impuesto el $1^{\circ}$ de septiembre de 2019 , a $7.5 \%$. Asimismo, para otros productos cuyo valor total es de $\$ 250$ mil millones de dólares se mantiene la tasa de $25 \%$.

Por parte de China, además de realizar ajustes en el manejo de divisas, de la protección de propiedad intelectual, de la reforma financiera, etc., se comprometió a incrementar las compras de productos estadounidenses en un monto de $\$ 200$ mil millones de dólares, considerando las importaciones de 2017 como la base, en un lapso de dos años desde el $1^{\circ}$ de enero de 2020

8. La Asociación Económica Integral Regional (RCEP, por sus siglas en inglés) es un acuerdo de libre comercio firmado en la Cumbre de la Asociación de Naciones del Sudeste Asiático (ASEAN, por sus siglas en inglés) integrada por Birmania, Brunei, Camboya, Filipinas, Indonesia, Laos, Malasia, Singapur, Tailandia y Vietnam, que se celebró el 15 de noviembre de 2020 con cinco Estados de Asia y Oceanía (Australia, China, Corea del Sur, Japón y Nueva Zelanda), se espera que entre en vigor en un plazo menor a dos años.

Cabe mencionar que este acuerdo de libre comercio con 15 países miembros se convierte en la región más grande del mundo, superando a los de la norteamericana y de la europea, con aproximadamente $30 \%$ de la población mundial y del producto mundial bruto, respectivamente. 
hasta el 31 de diciembre de 2021, distribuidas en productos farmacéuticos, energéticos, agrícolas y de servicios.

Por todo lo anterior se puede decir que el entorno externo se torna cada vez más complejo y que los factores adversos han generado un alto grado de incertidumbre para el crecimiento económico de China, por lo que este país enfrenta la necesidad de hacer un cambio a su modelo de desarrollo económico.

Para poder llevar a cabo el cambio, es condición sine qua non contar con un mercado interno fuerte y un consumo doméstico robusto, pues de una u otra manera el debilitamiento o la reducción de la demanda internacional podrían verse compensados por las necesidades crecientes y diversas generadas en el mercado interno. El análisis anterior, relacionado con el cambio observado en la importancia relativa del consumo doméstico, el sector terciario y del intercambio comercial dentro del PIB, de alguna manera confirma que el tiempo histórico ya llegó para China.

\subsection{Preparándose para el mediano y largo plazos: XIV PQDEYS 2021-2025}

El año 2020 ha sido crucial no solamente porque fue un año previo al cumplir el primer centenario de la fundación del PCCh, sino porque finalizó el XIII PQDEYS 2016-2020. Por ello, al arrancar 2021 la coincidencia entre el comienzo de una edición más del plan quinquenal y la instrumentación de un esquema renovador del crecimiento económico marcará el inicio de una nueva era del desarrollo económico chino.

De momento, a pesar de que todavía no se ha revelado el contenido del XIV PQDEYS 2021-2025, se podrían deducir los principales objetivos; a saber: en primer lugar, el periodo 2021-2050 se contemplará en dos fases, según lo establecido en el XIX Congreso Nacional del PCch inaugurado el 18 de octubre de 2017. En el primer subperiodo 2021-2035, el país construirá los cimientos de una sociedad modestamente acomodada, cuya finalidad es asegurar la creación básica de la modernización socialista. En los siguientes 15 años (2036-2050) el país seguirá trabajando para lograr plenamente la modernización socialista (Qiu, 2017).

En segundo lugar, Xi Jinping, en un discurso para explicar con detalles las propuestas del Comité Central del PCCh para formular el XIV PQDEYS 2021-2025 y los objetivos a largo plazo para 2035 a principios de noviembre de 2020, mencionó que la economía china podría duplicar su tamaño para 
2035 y que el país podría alcanzar el estatus de país de altos ingresos en los próximos cinco años (Xinhua, 2020b).

Lo anterior indicaría que en un lapso de 15 años se duplicará el tamaño de la economía desde una base de 101 billones de RMB aproximadamente. Por lo que se requeriría que la tasa del crecimiento económico anual fuera de 4.8\% en promedio desde 2021 a 2035. Asimismo, el PIB per cápita podría ubicarse en alrededor de 28,000 dólares equivalentes a precios constantes de 2020, considerando el ajuste cambiario y el crecimiento demográfico, cifra correspondiente al mediano de los países dentro del grupo del ingreso alto definido por el Banco Mundial. ${ }^{9}$

Cabe mencionar que para los 15 años futuros se espera que el crecimiento económico pueda seguir el mismo comportamiento que el registrado durante los últimos 10 años. De tal manera, para el periodo del XIV PQDEYS 2021-2025 la tasa del crecimiento económico probablemente se ubicaría en su nivel más alto que el promedio para los siguientes 15 años. Considerando que de 2016 a 2019 la economía china creció con una tasa promedio anual de 6.7\%, y bajó con un punto porcentual aproximadamente en comparación con la registrada de 2010 a 2015, se podría proyectar una tasa de crecimiento para el periodo 2021-2025 en un rango de 5.5-6.0\%, y para los próximos 15 años alcanzaría una cifra de $4.8 \%$ en promedio, ambos en términos anualizados reales. En caso de que se cumplieran los pronósticos anteriores, el PIB per cápita podría ubicarse en un rango de entre 14,017 y 14,354 dólares al finalizar 2025, ${ }^{10}$ con lo que superaría el umbral de los países de ingresos medios, fijado por el Banco Mundial en 2019, de 12,535 dólares (Serajuddin \& Hamadeh, 2020).

Con propósito de alcanzar la meta planteada en el XIV PQDEYs 2021-2025, las autoridades chinas podrían incluir las siguientes acciones concretas:

Promover el desarrollo y la innovación de ciencia y tecnología, para que alcance la "autosuficiencia tecnológica de producción nacional", sobre todo

9. De acuerdo con las proyecciones realizadas por un grupo de expertos de la Renmin University of China, la tasa del crecimiento económico necesitaría alcanzar un $4.8 \%$ en los 15 años futuros para que el PIB per cápita se duplique al terminar 2035 (Liu, 2020).

10. Los pronósticos consisten en las siguientes bases de la economía china: 1. En 2019, el PIB per cápita es de 70,892.0 RMB de acuerdo con las cifras publicadas por la oficina estadística de China; 2. En caso de que la tasa del crecimiento económico coincidiera con los pronósticos realizados por el Fondo Monetario Internacional, de 2\%, la cifra anterior alcanzaría 72,309.8 RMB para 2020. 3. La tasa del crecimiento demográfico seguirá con 0.4\% anualizada, la misma registrada de 2010 a 2019; 4. Al mismo tiempo, el tipo de cambio RMB-dólar se mantendría con 6.6:1.0. 
en las áreas claves y sustanciales en las cadenas de valor de producción y del suministro.

Enfatizar la importancia de un esquema renovado del crecimiento económico, basado en el mercado doméstico complementado por el global, de tal manera que se dé una interacción entre los dos tipos de mercado.

Impulsar aún más el proceso de apertura económica de China y su integración a la mundial, para que los recursos productivos tanto nacionales como internacionales puedan asignarse de acuerdo con las fuerzas de la libre competencia dentro de los mercados domésticos y globales integrados.

Mejorar aún más la competitividad y el marco regulatorio para prevenir los riesgos financieros y económicos.

Con base en estas acciones, se puede esperar que el cambio del modelo de crecimiento económico interno-externo sea dependiente del consumo interno como el medular. Si se concreta este acoplamiento de ambas orientaciones del mercado interno-externo en un futuro cercano, lo esencial sería articular de manera eficiente y con plenitud los distintos sectores y diferentes actividades económicas al interior del país, y de tal manera potenciaría aún más la fortaleza del mercado doméstico y del consumo nacional en su contribución al crecimiento económico.

A través de esa estrategia de "circulación dual" y de la interacción de los dos tipos de mercado, se espera que la economía china reciba un impulso adicional y en consecuencia mejorar aún más las perspectivas. Sin duda esto no solamente brindaría oportunidades de obtener una tasa mínima requerida del crecimiento económico, sino también significaría un empujón más a la recuperación de la economía mundial, que ha enfrentado una debacle profunda causada por la pandemia de la Covid-19 en 2020.

\section{Implicaciones en el cambio del modelo de desarrollo y crecimiento económico}

El modelo de desarrollo económico que implementó China desde hace más de 40 años fue exitoso desde finales del siglo xx y durante la primera década del xxI. Sin embargo, ante el cambiante entorno e incertidumbre creciente de la economía global, lo mismo ha registrado deficiencias en la sustentabilidad debido a la dependencia excesiva del mercado internacional y por el uso intensivo de los factores productivos. 
Por ello, China empezó a realizar ajustes graduales desde 2012, cuando enfatizó la importancia de buscar la calidad en lugar de la cantidad del crecimiento económico y al mismo tiempo planteó la necesidad de transformar el modelo de desarrollo económico desde uno basado en el mercado externo, por otro orientado al mercado interno como medular. Esto implica no solamente ajustes importantes para la economía china, sino también para el resto del mundo, debido a las siguientes consideraciones:

En primer lugar, el cambio en el modelo de desarrollo y crecimiento económico de ninguna manera significa que China ya no seguiría el mismo proceso de integrar su economía y mercado a los globales. Al contrario, las acciones han confirmado más que nunca su compromiso y su consistencia de llevar a cabo las reformas económicas y apertura comercial con mayor profundidad y amplitud.

En segundo lugar, se puede esperar que las mismas políticas derivadas del esquema renovado del modelo de desarrollo y crecimiento económico generarán una disminución paulatina del saldo favorable de China en sus intercambios comerciales con el resto del mundo, ante la necesidad de ese país para satisfacer las crecientes y diversas demandas en un mercado cada vez más dinámico y fortalecido. En este sentido, el cambio del modelo de crecimiento económico implicaría una gran oportunidad de negocios potenciales para el resto del mundo.

En tercer lugar, a pesar de lo anterior, el ajuste del modelo económico de ninguna manera causaría efectos negativos para la economía, pues la fortaleza y la potencialidad del mercado interno podrían compensar la parte perdida por los mercados externos. Una muestra de ello es que, ante las fricciones comerciales sostenidas con Estados Unidos desde mediados de 2018, la economía china ha podido solventar los impactos dañinos sin grandes altibajos. En este sentido, aunque la nueva administración de EU (periodo, 2021-2025) podría seguir manteniendo el mismo nivel arancelario para las importaciones de los productos originarios de China, los resultados ya están descontados y los podrá manejar con cierta flexibilidad y un gran margen de maniobra.

En otras palabras, la nueva estrategia del crecimiento económico basada en la circulación dual tiene el propósito de fortalecer el mercado interno y el consumo doméstico, y al mismo tiempo no debe subestimar la importancia de mantener el equilibrio de los flujos interrelacionados entre el comercio exterior y el interior. 
Finalmente, las mismas políticas de apertura económica y de integración a la global le permitirían a China buscar nuevas alianzas estratégicas para impulsar el comercio internacional y el flujo de IED. Sobre todo, formar parte de las nuevas cadenas de valor de acuerdo con las necesidades de su mercado interino. En este aspecto, la firma de China del RCEP, y la respuesta positiva de las autoridades de esa nación de participar en las negociaciones con los miembros del Tratado Integral y Progresista de Asociación Transpacífico (TIPAT; CPTPP, por sus siglas en inglés), son ejemplos evidentes de esa dirección.

\section{Referencias bibliográficas}

An, Y. (2016). Reformas del lado de oferta. https://baike.baidu.com/item/\%E 4\%BE\%9B\%E7\%BB\%99\%E4\%BE\%A7\%E7\%BB\%93\%E6\%9E\%84\%E6\% 80\%A7\%Е6\%94\%B9\%Е9\%9D\%A9

Barone, B. (2015). El debate sobre la condición de economía de mercado de China cobra intensidad (DGEXPO/B/PolDep/Note/2015_330). Departamento Temático, Dirección General de Políticas Exteriores, European Parliament. http://www.europarl.europa.eu/RegData/etudes/IDAN/2015/570453/ EXPO_IDA(2015)570453_ES.pdf

Consejo Estatal de China. (2020). 国务院关于印发北京、湖南、安徽自由 贸易试验区总体方案及浙江自由贸易试验区扩展区域方案的通 知Ishii Kashiwazaki Kariwa Genshiryokuhatsuden- jochou Teirei Taiken [Anunciamiento de extender zonas del libre comercio para Beijing, Hunan y Anhui] (número 10). http://www.gov.cn/zhengce/content/2020-09/21/ content_5544926.htm

Fondo Monetario Internacional (FMI). (2015a, septiembre 30). IMF's Executive Board Completes Review of SDR Basket, Includes Chinese Renminbi [Press Release No. 15/540]. https://www.imf.org/en/News/Articles/2015/09/14/01/49/pr15540

Fondo Monetario Internacional. (2015b, diciembre 1). IMF Executive Board Completes the 2015 Review of SDR Valuation [Press Release No. 15/543]. https://www.imf.org/en/news/articles/2015/09/14/01/49/pr15543

Fondo Monetario Internacional. (2020). Capítulo 1: Perspectivas y Políticas Mundiales. En Fondo Monetario Internacional, Perspectivas de la economía mundial. Fondo Monetario Internacional. https://www.imf.org/es/ Publications/WEO/Issues/2020/09/30/world-economic-outlook-october2020\#Capitulo1 
Fondo Monetario Internacional. (2021). Actualización de las perspectivas de la economía mundial, enero de 2021. Informes de perspectivas de la economía mundial: enero de 2021. https://www.imf.org/es/ /link.aspx?_id=B52E 2E0927854FC8823D98E147138A43\&_z=z

González, J. G. (2002). La aceptación regreso de China a la OMC (Cuaderno de Trabajo). El Colegio de México.

González, J. G., \& Liu, X. (2019). Retos socioeconómicos del sueño chino en el primer cuarto del siglo xxi. En I. Perrotini \& J. A. Vázquez (Coords.), Alternativas de política monetaria en la poscrisis pp. 259-278. Universidad Nacional Autónoma de México.

Li, K. (2013, noviembre 13). Li Keqiang's report on the economic situation at the 16th National Congress of ACFTU (the All-China Federation of Trade Unions). China.org.cn http://www.china.org.cn/chinese/2013-11/13/ content_30582185.htm

Liu, X. (2017). Desincorporación del suelo colectivo y el sistema urbano en China 1990-2015. Problemas del Desarrollo. Revista Latinoamericana de Economía, 48(189), 169-188. https://doi.org/10.22201/ iiec.20078951e.2017.189.57546

Liu, X. (2019a). Cooperación económica bilateral entre China y México: reformas desde el enfoque de oferta. En A. Vargas, A Girón, G. Pulido \& L. Jian (Coords.), China y México: 45 años de relaciones diplomáticas y culturales (pp. 129-136). Universidad Nacional Autónoma de México.

Liu, X. (2019b, octubre 29-30). Fricciones comerciales de China-los Estados Unidos y sus impactos sobre el crecimiento económico para México [Ponencia]. Seminario 70 años de existencia de la República Popular de China, 19492019. Ciudad de México, México.

Liu, X., Zhang, Q., Chen, F., Bo, W., Husheng, A., \& Yan, Y. (2015). La reestructuración económica y las nuevas estrategias del desarrollo en China (Cuadernos de Trabajo del Chechimex, número 1). Centro de Estudios China-México, Facultad de Economía, UNAM. https://dusselpeters.com/CECHIMEX/ Revista_Cchmx_1_2015_.pdf

Liu, Y. (2020, noviembre 13). 刘元春：科学测算和设定2035和 “十四五” 经济增长目标 [Fijar la meta del crecimiento económico para el periodo de 2021-2035 y de 14vo plan quinquenal a través de cálculos científicos]. Sina Finance. http://finance.sina.com.cn/zl/china/2020-11-12/zliiznctke1109286.shtml?cre=zl\&r=user\&pos=3_3 
Ministerio del Comercio de China. (2020, agosto 12). Aviso del proyecto integral de innovación y desarrollo en el sector de servicios. http://www.gov.cn/ zhengce/zhengceku/2020-08/14/content_5534759.htm

National Bureau of Statistics of China. (2020a). China Statistical Yearbook 2019. http://www.stats.gov.cn/tjsj/ndsj/2019/indexch.htm

National Bureau of Statistics of China. (2020b). Reporte anual del desarrollo socioeconómico 2019. http://www.stats.gov.cn/tjsj/zxfb/202002/ t20200228_1728913.html

National Bureau of Statistics of China. (2021). Reporte anual del desarrollo socioeconómico para 2020. http://www.stats.gov.cn/tjsj/zxfb/202102/ t20210227_1814154.html

Qiu, Xi. (2017, octubre 19). Luchar por el cumplimiento de los dos objetivos centenarios. El Financiero. https://www.elfinanciero.com.mx/opinion/ qiu-xiaoqi/luchar-por-el-cumplimiento-de-los-dos-objetivos-centenarios

Ríos, X. (2016). El XIII Plan Quinquenal: antecedentes, contexto, contenidos y expectativas. Araucaria. Revista Iberoamericana de Filosofía, Política y Humanidades, 18(35), 231-247. https://dx.doi.org/10.12795/araucaria.2016.i35.12

Serajuddin, U., \& Hamadeh, N. (2020, julio 01). Nuevas clasificaciones de los países elaboradas por el Banco Mundial según los niveles de ingreso: 20202021. Banco Mundial Blogs. https://blogs.worldbank.org/es/datos/ nuevas-clasificaciones-de-los-paises-elaboradas-por-el-banco-mundialsegun-los-niveles-de-ingreso

Sina Finance. (2014, noviembre 10). 习近平首次明确概括经济新常态 [Xi Jingping conceptualizó la nueva normalidad económica por primera vez]. Sina Finance. http://finance.sina.com.cn/china/20141110/023020771827. shtml

The State Council Information Office of the People's Republic of China. (2018). 《中国与世界贸易组织》白皮书（全文 [Libro Blanco “China y la Organización Mundial del Comercio]. http://www.scio.gov.cn/zfbps/32832/ Document/1632334/1632334.htm

Villarreal, R. (1988). Industrialización, deuda y desequilibrio externo de México: Un enfoque neoestructuralista (1929-1988). (2da edición). Fondo de Cultura Económica.

Xinhua. (2017, noviembre 3). Texto íntegro del informe presentado por Xi Jinping ante XIX Congreso Nacional del PCCh. http://spanish.xinhuanet.com/201711/03/c_136726335.htm 
Xinhua. (2020a, julio 28). Texto íntegro: Intervención del Presidente Xi Jinping en la inauguración de la Quinta Reunión Anual del Banco Asiático de Inversión en Infraestructuras. http://spanish.xinhuanet.com/202007/28/c_139246500.htm

Xinhua. (2020b, noviembre 3). La economía china es prometedora y tiene potencial para mantener desarrollo estable a largo plazo, afirma Xi. http://spanish. xinhuanet.com/2020-11/03/c_139488147.htm

Xinhua en VI Magazine. (2020, octubre 20). Enfoque: Comprendiendo la «circulación dual» de China y sus implicaciones para el mundo. https://xinhua. vimagazine.com.mx/2020/10/20/enfoque-comprendiendo-la-circulacion-dual-de-china-y-sus-implicaciones-para-el-mundo/ 